\title{
Systemic inflammation, genetic susceptibility and lung function
}

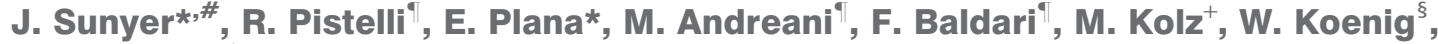 \\ J. Pekkanen ${ }^{f}$, A. Peters ${ }^{+}$and F. Forastiere**
}

ABSTRACT: Local inflammation in airway diseases is well recognised, but less is known about the association between low-grade systemic inflammatory processes and lung function. The aim of the present study was to assess the association between inflammatory markers and lung function, taking into account polymorphisms in genes coding for inflammatory markers.

In 134 post-myocardial infarction patients, six repeated measurements of $\mathrm{C}$-reactive protein (CRP), interleukin (IL)-6 and fibrinogen in peripheral blood were assayed using high-sensitivity tests. Spirometry was conducted at baseline. Genotyping of single nucleotide polymorphisms was performed in genes coding for the inflammatory markers.

CRP and IL-6 levels were negatively associated with forced expiratory volume in one second (FEV 1 ), forced vital capacity (FVC) and mean forced expiratory flow between 25 and $75 \%$ of FVC (FEF25-75\%). In the CRP gene, both the polymorphism rs1205 and the haplotype 2 showed a

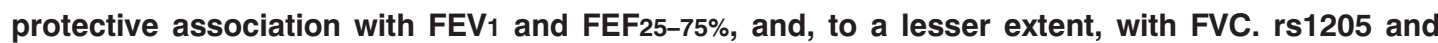
haplotype 2 were both negatively associated with CRP levels in peripheral blood. Analysis with instrumental variables also showed a protective effect between these CRP gene polymorphisms and lung function.

Results are very suggestive that heritability of lung function is at least partly controlled by the CRP gene. Applying a Mendelian randomisation approach, the study supports a causal association between low-grade general inflammation and airway diseases.

KEYWORDS: C-reactive protein, genes, lung function, Mendelian randomisation

$\mathbf{T}$ he local inflammatory process in the pathogenesis of chronic obstructive pulmonary disease (COPD) is well recognised

[1]. However, less is known about the low-grade systemic inflammatory process in COPD [2]. Many studies in the general population have reported higher levels of C-reactive protein (CRP) and fibrinogen in peripheral blood of individuals with impaired lung function [3-9] and in patients suffering from COPD [10-12]. Such associations may lead to the suggestion that pharmaceutical agents that lower CRP and fibrinogen levels could lower the risk of COPD. However, reverse causality cannot be excluded given that most studies conducted to date are cross-sectional, and a recent longitudinal study did not find an effect of CRP on decline in lung function over 9 yrs [9]. That study contradicts a previous longitudinal study, which showed that fibrinogen levels, 5 yrs previously, had moderately predicted lung function [13]. This latter finding might suggest that low-grade systemic inflammation could be crucial to the inflammatory process in the airways, parenchyma and pulmonary vasculature. However, these proteins were also associated with several factors that could confound the observed association, such as the occurrence of cardiovascular diseases. One approach towards avoiding residual confounding and reverse causation is the use of Mendelian randomisation [14]. In this approach, genotypes associated with acute phase proteins are directly related to the outcome. Very few studies have explored associations between genetic predisposition for an exaggerated inflammatory response and the risk of deterioration in pulmonary function [15]. The aim of the present study was to assess the association between inflammatory markers and lung function, taking into account polymorphisms in genes coding for these inflammatory markers.

\section{METHODS}

In a multicentre study of myocardial infarction (MI) survivors (the AIRGENE study), repeated samples were collected to measure inflammatory markers; in one of the study centres (Rome, Italy), lung function was measured. In total, 134 post-MI patients were recruited, on average, $2.7 \mathrm{yrs}$ after

\section{AFFILIATIONS}

${ }^{*}$ Centre for Environmental

Epidemiological Research and

\#Municipal Institute of Medical

Research, Universitat Pompeu Fabra,

CIBERESP, Barcelona, Spain.

'Catholic University and

**Local Health Authority, Rome, Italy.

${ }^{+}$GSF-National Research Centre for Environment and Health, Institute of Epidemiology, Neuherberg and $\S$ University of Ulm Medical Center, Ulm, Germany.

${ }^{f}$ National Public Health Institute (KTL), Helsinki, Finland.

CORRESPONDENCE

J. Sunyer

Centre for Environmental

Epidemiological Research

Municipal Institute of Medical

Research

Dr Aiguader 88

08003 Barcelona

Spain

Fax: 34933160575

E-mail: jsunyer@imim.es

Received:

May 032007

Accepted after revision:

March 172008

SUPPORT STATEMENT

The AIRGENE study was funded as part of the European Union's 5th Framework Programme, key action number 4: "Environment and Health", contract number QLRT2002-02236.

STATEMENT OF INTEREST None declared.

European Respiratory Journal Print ISSN 0903-1936 Online ISSN 1399-3003 
the last MI. Six repeated clinical examinations were scheduled, one every 4 weeks, providing a total of 741 blood samples. Patients with chronic inflammatory diseases were excluded. Each clinical visit was scheduled on the same time and day of the week. If patients suffered from acute infections, examinations were postponed. At each visit, an EDTA-plasma sample was collected and centrally assayed for CRP, fibrinogen and interleukin (IL)-6 using a nephelometre (BN-II; Dade-Behring, Deerfield, IL, USA) and a high-sensitivity colorimetric sandwich ELISA (R\&D Systems, Wiesbaden, Germany). Baseline questionnaires and a shorter instrument at each follow-up visit were administered by interview. Blood pressure, height and weight, N-terminal pro-B-type natriuretic peptide (NTproBNP), cholesterol and glycosylated haemoglobin were measured at baseline. All participating centres received approval of the study protocol by their local human ethics committees. Informed consent was obtained from all patients at the first clinical visit after a detailed description of the study protocol.

Lung function was measured at baseline following the American Thoracic Society/European Respiratory Society standard procedure [16]. Forced vital capacity (FVC), forced expiratory volume in the first second (FEV1) and mean forced expiratory flow between 25 and 75\% of FVC (FEF25-75\%) were selected as the outcome measurements. FEF25-75\% was chosen in order to have a measurement of bronchial obstruction that was more sensitive and independent of the expiratory effort than FEV1.

Genotyping of 36 polymorphisms was performed in the genes for CRP, IL-6 and fibrinogen (FGA, FGB and FGG) [17]. The SeattleSNPs Program for Genomic Applications database [18] was used for the selection of single nucleotide polymorphisms (SNPs). SNPs were chosen on the basis of positional and functional aspects to enhance the chance of detecting associations. PCR primers were designed by Sequenom's MassARRAY ${ }^{\mathrm{TM}}$ Assay Design program (Sequenom, San Diego, CA, USA), and then aligned to the gene cluster in order to check for accuracy and to eliminate false results from genotyping repeat regions [19]. Genotyping was performed using the MassARRAY ${ }^{\mathrm{TM}}$ system (Sequenom), as described previously [20]. To reduce the problem of multiple comparisons, haplotypes were assessed. Haplotypes have previously been reconstructed within blocks of high-linkage disequilibrium using expectation-maximisation algorithm and following the reading direction of the gene [20]. Each SNP was tested for departures from Hardy-Weinberg equilibrium by a Chi-squared test or Fisher's exact test depending on allele frequency $(p>0.05)$. None of the SNPs showed deviations from Hardy-Weinberg equilibrium.

Linear regression models were applied to evaluate the associations between FEV1, FVC and FEF25-75\% (as the outcomes), and inflammatory marker levels and SNPs, after adjusting for confounders including smoking, age, sex, height and body mass index. Variables such as NT-proBNP, cholesterol and glycosylated haemoglobin were also treated as confounders. For each subject, the mean of the repeated measures of inflammatory markers was calculated. Levels of inflammatory markers were treated both continuously (after $\log$ transformation) and categorised in tertiles to assess the linearity of relationships. The most common homozygote of each SNP was used as a reference group. Instrumental variables methods [21] were applied to obtain estimates of the unconfounded association between CRP and lung function, using the genetic variables as predictors of CRP levels in a system of two simultaneous equations. This method takes into account the association between genotypes and CRP levels as well as the relationship between CRP levels and lung function, and has been previously applied to the Mendelian randomisation approach [14, 22]. Mendelian randomisation assumes that the inheritance of common polymorphisms in genes associated with circulating levels of acute-phase proteins should be randomly allocated in maternal and paternal alleles at the time of gamete formation, according to Mendel's second law; therefore, the association obtained under instrumental variables could not be confounded. Comparison of linear regression and instrumental variable regression results was carried out using the Durbin-Wu-Hausman test [21].

\section{RESULTS}

Lung function values as well as subjects' characteristics and inflammatory markers are shown in table 1. CRP was negatively associated with all three lung function indicators, using the inflammatory markers in tertiles and as continuous variables (table 2). IL-6 was also associated with FEV1 and FVC, with significant associations with the highest tertile or using a continuous variable. These associations were not confounded by smoking characteristics or by variables related with MI severity (data not shown). Associations with fibrinogen were not statistically significant.

Among polymorphisms in CRP and $I L-6$ genes, only SNPs in the CRP gene showed an association with lung function measures. The rare homozygote alleles of the SNPs rs1205 and

\begin{tabular}{|c|c|c|}
\hline \multirow[t]{2}{*}{ TABLE 1} & \multicolumn{2}{|c|}{$\begin{array}{l}\text { Description of lung function, characteristics and } \\
\text { nflammatory markers }\end{array}$} \\
\hline & Males $^{\#}$ & Females \\
\hline FEV 1 L & $2.95 \pm 0.71(1.31-4.96)$ & $2.21 \pm 0.67(0.85-3.55)$ \\
\hline FVC L & $3.93 \pm 0.78(2.11-5.82)$ & $2.82 \pm 0.74(1.23-4.27)$ \\
\hline FEF $25-75 \%$ L & $2.53 \pm 1.20(0.44-6.89)$ & $2.15 \pm 1.11(0.49-4.25)$ \\
\hline Age yrs & $63.03 \pm 8.69(39-79)$ & $61.39 \pm 11.62(42-80)$ \\
\hline Height $\mathrm{cm}$ & $170.47 \pm 6.92(154-190)$ & $156.78 \pm 8.00(145-172)$ \\
\hline Weight kg & $79.65 \pm 12.51(47-113)$ & $66.78 \pm 16.57(42-106)$ \\
\hline BMI $\mathbf{k g} \cdot \mathrm{m}^{-2}$ & $27.37 \pm 3.76(15-39)$ & $27.15 \pm 6.14(15-39)$ \\
\hline Pack-yrs & $1.19 \pm 1.34(0.0-8.6)$ & $0.66 \pm 0.78(0.0-2.9)$ \\
\hline \multicolumn{3}{|l|}{ Smoking status } \\
\hline Current & $11(9.5)$ & $1(5.6)$ \\
\hline Ex-smoker $<5$ yrs & $49(42.2)$ & $9(50.0)$ \\
\hline Ex-smoker $>5$ yrs & 27 (23.3) & $2(11.1)$ \\
\hline Never & $29(25.0)$ & $6(33.3)$ \\
\hline Fibrinogen $\mathbf{g} \cdot \mathrm{L}^{-1}$ & $3.03(1.94-5.18)^{+}$ & $3.75(2.44-4.54)^{5}$ \\
\hline CRP $\mathrm{mg} \cdot \mathrm{L}^{-1}$ & $1.57(0.16-15.33)^{+}$ & $1.76(0.37-11.46)^{\S}$ \\
\hline $\mathrm{IL}-6 \mathrm{pg} \cdot \mathrm{mL}^{-1}$ & $2.25(0.95-61.37)^{+}$ & $3.19(1.04-6.42)^{\S}$ \\
\hline
\end{tabular}

Data are presented as mean \pm SD (range), $n(\%)$ or median (range). FEV 1 : forced expiratory volume in one second; FVC: forced vital capacity; FEF25-75\%: mean forced expiratory flow between 25 and $75 \%$ of FVC; BMI: body mass index; CRP: C-reactive protein; IL: interleukin. ${ }^{\#}: n=116 ;{ }^{\circ}: n=18 ;{ }^{+}: n=647 ;{ }^{\text {s: }} n=94$ 
TABLE 2 Change in lung function measurements for each increase in tertile of inflammatory markers

\begin{tabular}{|c|c|c|c|}
\hline & FEV 1 mL & FVC mL & FEF $25-75 \% \mathrm{~mL}$ \\
\hline \multicolumn{4}{|l|}{ Fibrinogen $\mathbf{g} \cdot \mathrm{L}^{-1}$} \\
\hline $2.9-3.5$ & $53.1(-158.6-264.8)$ & $52.1(-180.5-284.6)$ & $-21.5(-422.2-379.2)$ \\
\hline$>3.5$ & $-44.5(-278.0-188.9)$ & $-127.0(-383.5-129.5)$ & $1.7(-440.2-443.6)$ \\
\hline Log(Fibrinogen $)^{\#}$ & $-305.3(-778.3-167.8)$ & $-434.9(-955.3-85.5)$ & $-317.3(-1213.9-579.3)$ \\
\hline $1-2.6$ & $-166.6(-365.3-32.1)$ & $-331.9(-549.1--114.7)$ & $98.0(-280.4-476.3)$ \\
\hline$>2.6$ & $-344.3(-563.4--125.3)$ & $-378.0(-617.5--138.5)$ & $-423.9(-841.1--6.7)$ \\
\hline $\log (\mathrm{CRP})^{\#}$ & $-162.4(-256.2--68.6)$ & $-165.8(-270.1--61.4)$ & $-237.4(-417.7--57.2)$ \\
\hline \multicolumn{4}{|l|}{$\mathrm{IL}-6 \mathrm{pg} \cdot \mathrm{mL}^{-1}$} \\
\hline$<1.8$ & 0 (reference) & 0 (reference) & 0 (reference) \\
\hline $1.8-2.8$ & $7.6(-199.7-214.9)$ & $31.8(-197.5-261.0)$ & $-73.3(-469.4-322.7)$ \\
\hline
\end{tabular}

Data are presented as coefficient (95\% confidence interval) adjusted for age, sex, height, body mass index, time since smoking cessation and pack-yrs. FEV1: forced expiratory volume in one second; FVC: forced vital capacity; FEF25-75\%: mean forced expiratory flow between 25 and 75\% of FVC; CRP: C-reactive protein; IL: interleukin. \#: doubling of inflammatory markers' concentrations.

rs1800947 in the CRP gene were associated with better lung function (rs1205: p<0.01; rs1800947: nonsignificant; table 3).

Consistently the analysis of SNPs, haplotypes 2 and 5 in the CRP gene, tagged by the rs1205 and rs1800947 SNPs, were associated with greater levels of lung function markers (table 4). The association with haplotype 2 was statistically significant for FEV1 and FEF25-75\% $(\mathrm{p}<0.02)$. No association was seen for haplotypes in the IL-6 gene.

Larger reductions of lung function levels in relation to CRP levels were observed after applying an instrumental variable regression approach based on SNP rs1205 and haplotypes 2 and 5 in the CRP gene (table 5) as opposed to when linear

TABLE 3 Change in lung function measures for single nucleotide polymorphisms from the CRP gene

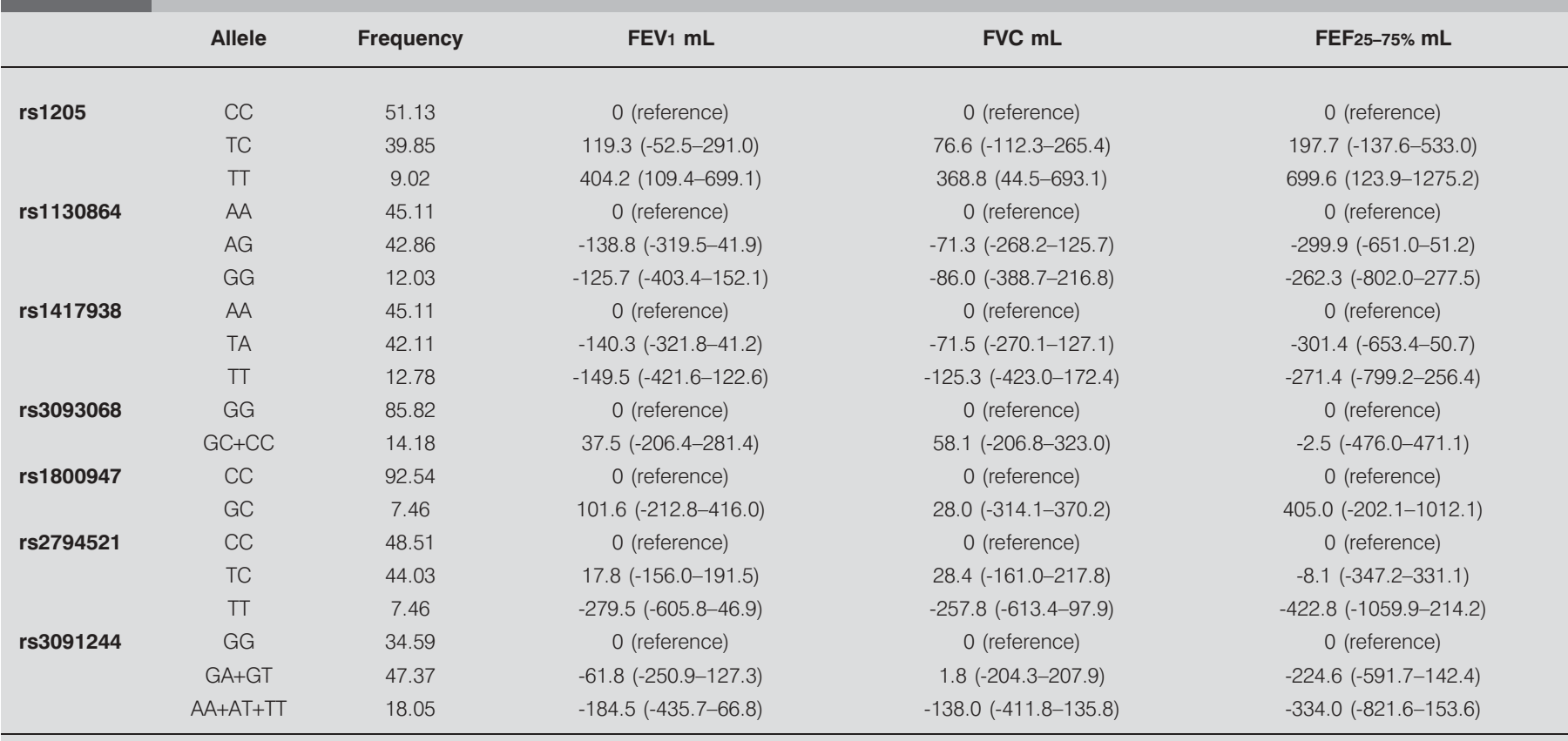

Data are presented as per cent or coefficient (95\% confidence interval) adjusted for age, sex, height, body mass index, unless otherwise stated. FEV1: forced expiratory volume in one second; FVC: forced vital capacity; FEF25-75\%: mean forced expiratory flow between 25 and $75 \%$ of FVC. 
TABLE 4 Change in lung function measures for haplotypes of CRP gene

\begin{tabular}{lcccc} 
& Frequency & FEV $\mathbf{~ m L}$ & FVC $\mathbf{~ L L}$ & FEF25-75\% $\mathbf{~ L L}$ \\
CR $\boldsymbol{P}^{\#}$ & & & & \\
TAACCG & 32.95 & 0 (reference) & 0 (reference) & 0 (reference) \\
TGTCTG & 24.62 & $182.3(32.4-332.3)$ & $145.5(-19.5-310.5)$ & $316.3(23.8-608.9)$ \\
CGTCCG & 29.17 & $26.6(-129.8-183.1)$ & $15.5(-156.7-187.6)$ & $57.3(-247.9-362.6)$ \\
TTTCCC & 7.58 & $70.3(-168.4-309.1)$ & $64.2(-198.5-326.9)$ & $123.0(-342.8-588.8)$ \\
TGTGTG & 3.41 & $166.8(-180.0-513.6)$ & $80.0(-301.7-461.6)$ & $515.4(-161.1-1192.0)$ \\
Rare & 2.27 & $238.9(-174.8-652.6)$ & $276.6(-178.7-731.8)$ & $232.5(-574.6-1039.7)$ \\
\hline
\end{tabular}

Data are presented as per cent or coefficient ( $95 \%$ confidence interval) adjusted for age, height, sex and body mass index. FEV1: forced expiratory volume in one second FVC: forced vital capacity; FEF25-75\%: mean forced expiratory flow between 25 and $75 \%$ of FVC. ${ }^{\#}$ : order of single nucleotide polymorphisms tagging the haplotype was rs2794521 rs3091244 rs1417938 rs1800947 rs1205 rs3093068; ${ }^{\bullet}$ : haplotypes with frequencies < <\%.

regression was used (table 2). However, the confidence intervals were larger. The generally low p-values obtained when comparing the two sets of test results suggests the models using instrumental variables are more suitable for these data than ordinary linear regression (table 5).

\section{DISCUSSION}

CRP and IL-6 peripheral levels were found to be associated with lung function. The CRP gene (the polymorphism rs1205 and the haplotype 2) was found to be associated with better lung function. rs1205 has been previously associated with lower levels of CRP in several studies [23-25]. rs1205 was strongly associated with lower baseline levels of CRP in the AIRGENE study including all individuals from the six study centres [26]. Better lung function was also found in individuals with haplotype 2 of the CRP gene. Haplotype 2 was related with lower levels of CRP in two previous studies [23, 27]. The present findings suggest that heritability of lung function, as well as of baseline CRP levels, is at least partly controlled by the CRP gene. Moreover, similar associations between rs1205 and haplotype 2 and lung function were found after using instrumental variables, which, according to the Mendelian randomisation approach, supports a primary association between low-grade general inflammation and airway diseases.

\begin{tabular}{|c|c|c|c|c|}
\hline \multirow[t]{2}{*}{ TABLE 5} & \multicolumn{4}{|c|}{$\begin{array}{l}\text { Change of lung function measures using the } \\
\text { single nucleotide polymorphism rs } 1205 \text { from the } \\
\text { CRP gene or the CRP haplotype } 2 \text { and } 5 \text { as } \\
\text { instrumental variables }\end{array}$} \\
\hline & rs1205 & p-value ${ }^{\#}$ & Haplotype 2-5 & p-value ${ }^{\neq}$ \\
\hline FEV1 & $-750.5(-1566.3-65.4)$ & 0.028 & $-666.1(-1391.5-59.3)$ & 0.051 \\
\hline FVC & $-628.0(-1402.8-146.8)$ & 0.108 & $-509.3(-1186.9-168.3)$ & 0.211 \\
\hline$F_{E F} 25-75 \%$ & $-1283.5(-2792.7-225.7)$ & 0.052 & $-1240.3(-2664.5-184.0)$ & 0.053 \\
\hline \multicolumn{5}{|c|}{$\begin{array}{l}\text { Data are presented as coefficient ( } 95 \% \text { confidence interval) for change (in } \mathrm{mL} \text { ) } \\
\text { with doubling the C-reactive protein level or p-value (test of equality between } \\
\text { ordinal linear regression and instrumental variables regression). FEV } 1 \text { : forced } \\
\text { expiratory volume in one second; FVC: forced vital capacity; FEF25-75\%: mean } \\
\text { forced expiratory flow between } 25 \text { and } 75 \% \text { of FVC. }{ }^{*} \text { : using the Durbin-Wu- } \\
\text { Hausman test. }\end{array}$} \\
\hline
\end{tabular}

The IL-6 gene was not associated with lung function, which might be explained by the local origin of IL-6. Finally, no association was found between fibrinogen levels and lung function, and accordingly no association was found between $F G A, F G B$ and $F G G$ genes and lung function.

An association between genotype and lung function provides evidence of a primary role of systemic inflammation in airway diseases based on the use of the Mendelian randomisation approach [14]. Mendelian randomisation has been used to discard causal relationships between acute-phase protein genes and metabolic syndrome [14], blood pressure [22] or a cardiac event [28]. In the present study, by contrast, an association between genotype and lung function was observed, suggesting a primary effect of low-grade inflammation on lung function. However, one limitation is the small study size, as reflected in the precision of the estimation of the associations between the SNP rs1205 and CRP in the present study sample $(\mathrm{F}=5, \mathrm{p}=0.01)$, in comparison with the greater precision when all AIRGENE study subjects were included $\left(p=10^{-8}\right)$. It is important to note that the association between rs1205 and CRP was homogeneous across all six centers in the AIRGENE study. However, the lack of power when using data from the single centre with available lung function measures might have biased the current estimations using instrumental variables [21]. Nevertheless, a potential causal association is supported by the following: no association was observed between lung function and the SNPs unrelated to CRP levels (table 3); after stratifying by smoking the association between $\mathrm{CRP}$ levels and lung function was stronger among neversmokers ( $p=0.01$ for interaction); the lack of any association between the rs1205 and the confounding variables ( $p>0.7)$. The frequency of the rs1205 allele varied largely in different countries. In the current study, $\sim 9 \%$ of subjects had the homozygote rare allele and $\sim 24 \%$ had haplotype 2 .

A stronger effect on FEF25-75\% than on FVC or FEV1 was observed. This could be due to the small size of the present study and random variation. However, given the internal consistency of the current results and the higher sensitivity of $\mathrm{FEF} 25-75 \%$ in detecting the bronchial obstruction at an early stage of the obstructive disease, this result could be explained by an early effect of genetic predisposition on the peripheral airways. 
A limitation in the present study is the small sample size, which decreases the possibility of finding significant associations with other SNPs in any of the genes examined. This could explain a lack of statistically significant associations with SNP rs1800947 in the CRP gene, as suggested by the haplotype analysis. The origin of the study population (MI survivors) might also explain the lack of associations with fibrinogen, given that almost all patients were undergoing statin treatment. In addition, the likelihood of surviving an MI may be dependent on respiratory comorbidity and on the genetic predisposition, which could affect the generalisability of the present results. However, the length of time elapsed since the last MI implies that individuals should have returned to baseline levels of CRP when they had blood samples taken in the present study. In addition, adjustment for variables related with MI severity and treatment did not confound the associations with lung function (data not shown). Moreover, the basis of the Mendelian randomisation approach lies in the fact that genetic variants will not generally be: liable to confounding by behavioural, socioeconomic and physiological factors; influenced by the onset of disease or by the tendency for individuals with disease to differentially report exposure history; and influenced by factors determining how participants are selected into a study, either as a case or a control [28]. In any case, the present study should be replicated in a general population.

The strength of the present study is the genotyping of the genes corresponding to inflammatory markers and the examination of their relationship to lung function given the lack of previous studies that have assessed these genes [15]. Another strength of the present study is the repetition of blood measurements in all participants, which reduced the common inter-subject variability to these biomarkers. A recent study on the effect of taking only a single measurement in a study of biomarkers has shown that, for CRP, using a single measurement could modify the regression coefficient by $40 \%$ [26]. Finally, potential limitations of the Mendelian randomisation approach, such as linkage disequilibrium, population stratification and pleiotropy [28], do not affect the polymorphisms investigated.

Overall, the present study strengthens the idea that systemic low-grade inflammation has a primary effect on lung function. However, due to the small size of the present study and the population origin, larger studies in general populations involving genes in systematic inflammatory process are required to replicate the present findings.

\section{REFERENCES}

1 Pawels R, Rabe K. Burden and clinical features of chronic obstructive pulmonary disease (COPD). Lancet 2004; 364: 613-620.

2 Sin DD, Man SF. Commentary: fuelling the fire-systemic inflammation and development of lung disease in the general community. Int J Epidemiol 2006; 35: 1008-1010.

3 Dahl M, Tybjaerg-Hansen A, Vestbo J, Lange P, Nordestgaard BG. Elevated plasma fibrinogen associated with reduced pulmonary function and increased risk of chronic obstructive pulmonary disease. Am J Respir Crit Care Med 2001; 164: 1008-1011.
4 Engström G, Lind P, Hedblad B, et al. Lung function and cardiovascular risk: relationship with inflammationsensitive plasma proteins. Circulation 2002; 106: 25552560.

5 Mannino DM, Ford ES, Redd SC. Obstructive and restrictive lung disease and markers of inflammation: data from the Third National Health and Nutrition Examination. Am J Med 2003; 114: 758-762.

6 Gan WQ, Man SF, Sin DD. The interactions between cigarette smoking and reduced lung function on systemic inflammation. Chest 2005; 127: 558-564.

7 Kony S, Zureik M, Driss F, Neurkirch C, Leynaert B, Neurkirch F. Association of bronchial hyperresponsiveness and lung function with C-reactive protein (CRP): a population based study. Thorax 2004; 59: 892-896.

8 Aronson D, Roterman I, Yigla M, et al. Inverse association between pulmonary function and C-reactive protein in apparently healthy subjects. Am J Respir Crit Care Med 2006; 174: 626-632.

9 Fogarty AW, Jones S, Britton JR, Lewis SA, McKeever TM. Systemic inflammation and decline in lung function in a general population: a prospective study. Thorax 2007; 62: 515-520.

10 Sin DD, Man SF. Why are patients with chronic obstructive pulmonary disease at increased risk of cardiovascular diseases? The potential role of systemic inflammation in chronic obstructive pulmonary disease. Circulation 2003; 107: 1514-1519.

11 Gan WQ, Man SF, Senthilselvan A, Sin DD. Association between chronic obstructive pulmonary disease and systemic inflammation: a systematic review and a metaanalysis. Thorax 2004; 59: 574-580.

12 VM Pinto-Plata, Müllerova $\mathrm{H}$, Toso JF, et al. C-reactive protein in patients with COPD, control smokers and nonsmokers. Thorax 2006; 61: 23-28.

13 Thyagarajan B, Jacobs DR, Apostol GG, Smith LJ, Lewis CE, Williams OD. Plasma fibrinogen and lung function: the CARDIA Study. Int J Epidemiol 2006; 35: 1001-1008.

14 Timpson NJ, Lawlor DA, Harbord RM, et al. C-reactive protein and its role in metabolic syndrome: mendelian randomisation study. Lancet 2005; 366: 1954-1959.

15 Yanbaeva DG, Dentener MA, Creutzberg EC, Wouters EF. Systemic inflammation in COPD: is genetic susceptibility a key factor? COPD 2006; 3: 51-61.

16 Miller MR, Hankinson J, Brusasco V, et al. Standardisation of spirometry. Eur Respir J 2005; 26: 319-338.

17 Peters A, Schneider A, Greven S, et al. Air pollution and inflammatory response in myocardial infarction survivors: gene-environment-interactions in a high-risk group. Inhal Toxicol 2007; 19: Suppl. 1, 161-175.

18 SeattleSNPS Variation Discovery Resource. http:/ /pga.gs. washington.edu/ Date last accessed: March 2006. Date last updated: March 2006.

19 BLAT Search Genome. http://genome.ucsc.edu/cgi-bin/ hgBlat Date last accessed: March 2006. Date last updated: March 2006.

20 Weidinger S, Klopp N, Wagenpfeil S, et al. Association of a STAT 6 haplotype with elevated serum IgE levels in a population based cohort of white adults. J Med Genet 2004; 41: 658-663. 
21 Martens EP, Pestman WR, de Boer A, Belitser SV, Klungel $\mathrm{OH}$. Instrumental variables. Aplications and limitations. Epidemiology 2006; 17: 260-267.

22 Davey Smith G, Lawlor DA, Harbord R, et al. Association of $\mathrm{C}$-reactive protein with blood pressure and hypertension: life course confounding and mendelian randomization tests of causality. Arterioscler Thromb Vasc Biol 2005; 25: 1051-1056.

23 Kardys I, de Maat MP, Uitterlinden AG, Hofman A, Witteman JC. C-reactive protein gene haplotypes and risk of coronary heart disease: the Rotterdam Study. Eur Heart J 2006; 27: 1331-1337.

24 Russell AI, Cunninghame Graham DS, Shepherd C, et al. Polymorphism at the C-reactive protein locus influences gene expression and predisposes to systemic lupus erythematosus. Hum Mol Genet 2004; 13: 137-147.
25 Miller DT, Zee RY, Suk Danik J, et al. Association of common CRP gene variants with CRP levels and cardiovascular events. Ann Hum Genet 2005; 69: 623-638.

26 Kolz M, Koening W, Müller M, et al. DNA variants, plasma levels and variability of C-reactive protein in myocardial infaction survivors: results from the AIRGENE study. Kongress Medizin und Gesellschaft, 2007. www.egms.de/ en/meetings/gmds2007/07gmds205.shtml.

27 Block G, Dietrich M, Norkus E, et al. Intraindividual variability of plasma antioxidants, markers of oxidative stress, C-reactive protein, cotinine, and other biomarkers. Epidemiology 2006; 17: 404-412.

28 Davey Smith G, Ebrahim S. What can mendelian randomisation tell us about modifiable behavioural and environmental exposures? BMJ 2005; 330: 1076-1079. 